\title{
Scalar resonances in leptophilic multi-Higgs-boson models
}

\author{
J. C. Montero, C. A. de S. Pires, and V. Pleitez \\ Instituto de Física Teórica, Universidade Estadual Paulista, Rua Pamplona 145, 01405-900 São Paulo, SP, Brazil
}

(Received 14 December 1998; published 8 September 1999)

\begin{abstract}
We show that the Higgs resonance can be amplified in a 3-3-1 model with a multi-Higgs-boson "leptophilic" scalar sector. This would allow the observation of the Higgs particle in muon colliders even for Higgs boson masses considerably higher than the ones expected to be seen in the electroweak standard model framework. [S0556-2821(99)02017-2]
\end{abstract}

PACS number(s): 12.60.Fr, 12.60.Cn, 13.10.+q, 14.80.Cp

The study of electroweak symmetry breaking is one of the main goals of future colliders. The Higgs boson coupling to fermions is proportional to the fermion mass and hence the $s$-channel Higgs contribution to $e^{+} e^{-} \rightarrow f \bar{f}$ is highly suppressed in electron-positron colliders. However, since muons are nearly 200 times heavier than electrons, it is usually considered in the literature that the $s$-channel production of the Higgs boson in the $\mu^{+} \mu^{-}$collider is one of the main features of muon colliders. The main process is also $\mu^{+} \mu^{-}$ $\rightarrow f \bar{f}[1]$.

We would like to point out that there exist a kind of model in which a scalar multiplet couples only with leptons (not with quarks). If this is the case, the vacuum expectation value (VEV) of the neutral component of such a multiplet can be naturally small since it is the only VEV responsible for generating the leptons masses. An example of this kind of model is the multi-Higgs-boson extensions of the electroweak standard model (ESM) with at least two Higgs doublets in such a way (say, by imposing an appropriate discrete symmetry) that one of them couples only with leptons in the fermion sector.

There are models in which such a situation arises in a more natural way than in the several doublet extension of the ESM. These are models based on $\mathrm{SU}(3)_{C} \otimes \mathrm{SU}(3)_{L} \otimes \mathrm{U}(1)_{N}$ gauge symmetry (3-3-1 model for short) [2]. In the minimal version of this model a sextet $(\mathbf{6 , 0})$ is necessary to give mass to the charged leptons and it does not couple to quarks indeed, so its VEV does not have to be necessarily a large VEV; say, it can be of the order of a few GeVs. A scalar sector having this particular characteristic will be called ' 'leptophilic.' Hence, leptophilic models can have a leptonHiggs-boson coupling stronger than the ESM one. This characteristic will be the key for having a Higgs resonanceenhancing effect in processes involving leptons in the initial and/or final state. Here we will consider $\mu^{+} \mu^{-}$collisions to study the Higgs resonance in the reactions $\mu^{+} \mu^{-}$ $\rightarrow W^{+} W^{-}, \mu^{+} \mu^{-}, b \bar{b}$ (WW, $\mu \mu, b b$, for short).

Throughout this work, for each process, we will refer to the pure Higgs contribution at $\sqrt{s}=m_{h}$ as signal $\left(\mathrm{S}=\sigma_{h}\right)$ and to the non-Higgs ones as background $\left(\mathrm{B}=\sigma_{\mathrm{B}}\right)$. Here we are also assuming that the pure 3-3-1 contributions to each reaction, i.e., the ones that are not contained in the ESM, are mediated by fields which are massive enough to make these contributions negligible. In this way the only difference between the 3-3-1 model and the ESM lies in the scalar sector so that the background will be the same for both models for each process considered. The background itself depends on the process: it is formed by the $\nu, \gamma$, and $Z$ contributions for $W W$ and by the $\gamma$ and $Z$ ones for $\mu \mu$ and $b b$.

Once the ratio $N_{\mathrm{S}} / \sqrt{N_{\mathrm{B}}}=\sqrt{\mathcal{L}_{\mu \mu}} \sigma_{h} / \sqrt{\sigma_{\mathrm{B}}}$ and $\mathcal{L}_{\mu \mu}$, the muon-collider luminosity, depends only on the machine, we focus our attention on the model-dependent dimensional ratio $\delta_{x}=\mathrm{S}_{x} / \sqrt{\mathrm{B}_{x}}=\left(\sigma_{h} / \sqrt{\sigma_{\mathrm{B}}}\right)_{x}(\mathrm{~cm})$, for $x=\mu \mu, b b, W W$. We are aware that the luminosity is a function of the beam energy: however, here we will consider a constant luminosity $\mathcal{L}_{\mu \mu}=10^{33} \mathrm{~cm}^{-2} \mathrm{~s}^{-1}=10 \mathrm{fb}^{-1} \mathrm{yr}^{-1}$ for energies in the range $100 \leqslant \sqrt{s} \leqslant 200 \mathrm{GeV}$.

In this work the integrated cross sections $\left(\sigma_{h}\right)$ are obtained by using a customized version of the COMPHEP package [3] so that the resonance condition is obtained by setting $\sqrt{s}=m_{h}$ as an input instead of using the usual Breit-Wigner expression [1]. We are also assuming a very high beam energy resolution so that the rms error $\left(\sigma_{\sqrt{s}}\right)$ will be much smaller than the total Higgs boson width $\left(\sigma_{\sqrt{s}} \ll \Gamma_{h}\right)$ and in this limit the effective cross section $\left(\bar{\sigma}_{h}\right)$, obtained by convoluting $\sigma_{h}(s)$ with the Gaussian distribution in $\sqrt{s}$, is equal to the integrated one $\left[\bar{\sigma}_{h}=\sigma_{h}\left(\sqrt{s}=m_{h}\right)\right]$. Although large polarization can enhance the ratio S/B [4], here we are not considering muon-beam polarization.

The total Higgs boson width is model dependent: however, we are assuming that for the Higgs boson mass range considered here (100-200 GeV) no new 3-3-1 decay modes are allowed leaving the total $\Gamma_{h}$ equal to the one of the ESM. In order to study the scalar resonance when comparing the 3-3-1 model with the ESM we will introduce the Higgssignal-enhancing factor (HSEF), here denoted by $f_{x}$ defined for each process by $\mathrm{S}_{x}^{331} / \mathrm{S}_{x}^{E S M}$.

The main goal of this paper is to show the possible existence of a Higgs-signal-enhancing effect due to the manifestation of the leptophilic quality of a given model; hence as we are not considering the experimental detection aspects in detail we assume that the detector efficiency is 1 .

Although we will consider explicitly a 3-3-1 model, we would like to stress that our results will be valid in multiHiggs-boson extensions of the ESM if they are implemented in such a way that there is only a doublet coupling to leptons as we said above.

In the 3-3-1 model considered here the lepton mass term transforms as $(\mathbf{1}, \mathbf{3}, 0) \otimes(\mathbf{1}, \mathbf{3}, 0)=\left(\mathbf{1}, \mathbf{3}^{*}, 0\right)_{A} \oplus(\mathbf{1}, \mathbf{6}, 0)_{S}$; then we can introduce a triplet $\eta=\left(\eta^{0}, \eta_{1}^{-}, \eta_{2}^{+}\right)^{T} \sim(\mathbf{1}, \mathbf{3}, 0)$ and a symmetric sextet $S_{H} \sim\left(\mathbf{1}, \mathbf{6}^{*}, 0\right)$. With the $\eta$ triplet only, one of the charged leptons remains massless and the two other 
are mass degenerate. Hence, the sextet $S_{H}$ at least has to be introduced in order to give arbitrary masses to all charged leptons. This scalar multiplet has the following charge attribution:

$$
S_{H}=\left(\begin{array}{ccc}
\sigma_{1}^{0} & h_{2}^{+} / \sqrt{2} & h_{1}^{-} / \sqrt{2} \\
h_{2}^{+} / \sqrt{2} & H_{1}^{++} & \sigma_{2}^{0} / \sqrt{2} \\
h_{1}^{-} / \sqrt{2} & \sigma_{2}^{0} / \sqrt{2} & H_{2}^{--}
\end{array}\right) .
$$

In the model, in order to give mass to the quarks, it is necessary to have two more triplets $\rho=\left(\rho^{+}, \rho^{0}, \rho^{++}\right)^{T}$ $\sim(\mathbf{1}, \mathbf{3}, 1)$ and $\chi=\left(\chi^{-}, \chi^{--}, \chi^{0}\right)^{T} \sim(1,3,-1)$.

Denoting the respective neutral field VEVs by $\mathrm{v}_{a}(a$ $=\eta, \rho, \chi)$ for the triplets and $\mathrm{v}_{S}$ for the $\sigma_{2}^{0}$ sextet field, and also assuming $\mathrm{v}_{\sigma_{1}}=0$, in such a way that neutrinos remain massless (assuming that the total lepton number is conserved), we can write the masses of the vector bosons:

$$
M_{W}^{2}=\frac{g^{2}}{4}\left(\mathrm{v}_{\eta}^{2}+\mathrm{v}_{\rho}^{2}+\mathrm{v}_{S}^{2}\right), \quad M_{Z}^{2} \approx \frac{g^{2}}{4 c_{W}^{2}}\left(\mathrm{v}_{\eta}^{2}+\mathrm{v}_{\rho}^{2}+\mathrm{v}_{S}^{2}\right) .
$$

Notice that $v_{\chi}$ does not contribute to the vector boson masses (it only contributes to the $Z$ boson mass but in such a way that it is proportional to $v / v_{\chi}$, with $v$ being any of the other VEVs). Since the $v_{\chi}$ is the VEV which is in control of the $\mathrm{SU}(3)_{L}$ breaking, it is larger than the other ones and we can neglect its contribution to $M_{Z}$. Hence, assuming that $v_{\chi}$ decouples at low energies, only three neutral scalars are important [5]; denoting by $h_{i}^{0}, i=1,2,3$, the mass eigenstates and $H_{a}^{0}, a=\eta, \rho, S$, the real part of scalar fields which are related by the orthogonal matrix $O, H_{a}^{0}=O_{a j} h_{i}^{0}$, and $\mathrm{v}_{a}$ denote the respective VEVs. In particular $H_{S}^{0}$ denotes the real part of $\sigma_{2}^{0}$. Since this field couples only with leptons its $\operatorname{VEV}\left(\mathrm{v}_{S}\right)$ may be of the order of a few $\mathrm{GeV}$.

The interaction of the $W$ boson with the scalars is $\mathcal{L}_{h W W}$ $=\left(g^{2} / 2\right) \vee_{a} O_{a j} h_{j}^{0} W^{+} W^{-}$, while the Yukawa interaction in the lepton sector is given by $\mathcal{L}^{Y}=-\left(m_{l} / \mathrm{v}_{S}\right) O_{S_{j}} h_{j}^{0} \overline{l l}$, since only the sextet $\left(H_{S}^{0}=O_{S_{j}} h_{j}\right)$ couples with them. Notice that this scalar interaction with leptons is not necessarily negligible since $\mathrm{v}_{S}$ can be of the order of some GeVs as commented earlier.

First we will study the $\mu^{+} \mu^{-} \rightarrow W^{+} W^{-}$process. There are four contributions to the $W W$ process, three of them being in the $s$ channel with $Z^{0}, A$ (photon), and $h^{0}$ 's exchanges and one in the $t$ channel with a neutrino exchange. In the 3-3-1 model all the $W$ couplings have the same form as in the standard electroweak model. The neutrino $M^{\nu}$ and the vector boson contributions $\left(M^{\gamma}, M^{Z}\right)$ are exactly the same as in the ESM. However, the scalar contributions are different in 3-3-1 models. Each $s$-channel exchange of a physical Higgs boson $h_{j}$ gives the contribution

$$
\begin{aligned}
\mathcal{M}^{h_{j}}= & \frac{4 i G_{F} m_{\mu} m_{W}^{2} O_{S_{j}}}{\sqrt{2} \mathrm{v}_{S}\left[\left(s-m_{j}^{2}\right)^{2}+m_{j}^{2} \Gamma_{j}^{2}\right]} \sum_{a} O_{a j} \mathrm{v}_{a}\left[\left(s-m_{j}^{2}\right)\right. \\
& \left.-i m_{j} \Gamma_{j}\right] \nabla\left(p_{2}, r_{2}\right) u\left(p_{1}, r_{1}\right) g_{\rho \sigma} \epsilon^{\rho} \epsilon^{\sigma},
\end{aligned}
$$

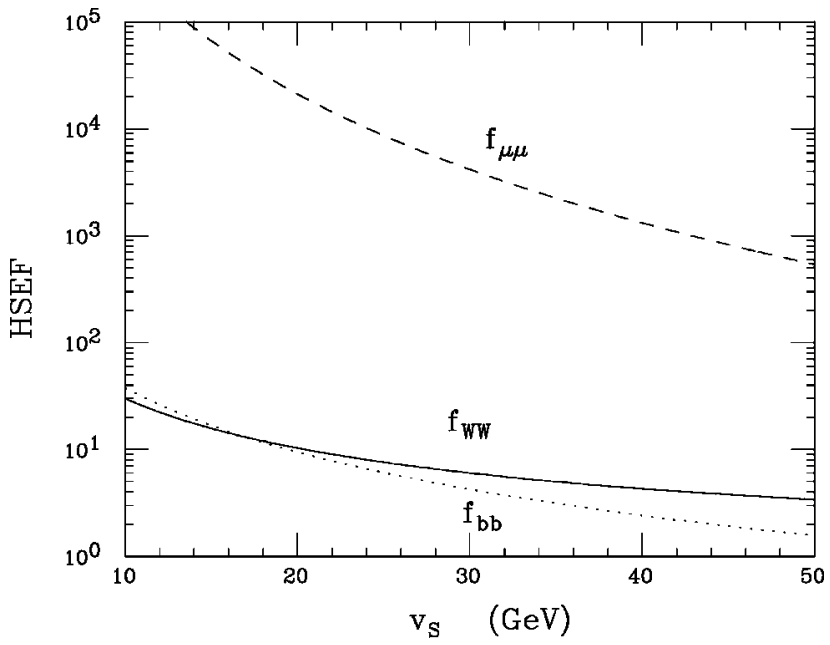

FIG. 1. The HSEF $f_{W W}$ (solid line), $f_{\mu \mu}$ (dashed line), and $f_{b \bar{b}}$ (dotted line) as a function of $\vee_{S}$ for $O_{\eta 3}=0.18, O_{\rho 3}=0.085, O_{S 3}$ $=0.98$, and for $\mathrm{v}_{\eta}=\mathrm{v}_{\rho}=\sqrt{\left(\mathrm{v}_{E S M}^{2}-\mathrm{v}_{S}^{2}\right) / 2}$.

where $m_{j}$ and $\Gamma_{j}$ are the mass and total width of the scalar $h_{j}^{0}$ (with no sum in $j$ ).

In the ESM a scalar resonance can only be revealed in a $\mu$ collider for a relatively light Higgs boson. This can be understood by the following simple reasoning. At the Higgs pole $\left(\sqrt{s}=m_{h}\right)$ the squared Higgs amplitude is proportional to $1 / m_{h}^{2} \Gamma_{h}^{2}$, where the total Higgs boson width $\Gamma_{h}$ is a fast growing function of the Higgs boson mass: it goes from $\sim 3.1 \times 10^{-3} \mathrm{Gev}$ for $m_{h} \sim 110 \mathrm{GeV}$ to $\sim 1.6 \mathrm{GeV}$ for $m_{h}$ $\sim 200 \mathrm{GeV}$. Hence the total Higgs contribution is suppressed for relatively large values of the Higgs boson mass. This argument is also valid for $\mu \mu$ and $b b$.

On the other hand, for a 3-3-1 model, as it can be seen from Eq. (3), the squared Higgs amplitude at $\sqrt{s}=m_{j}$ is proportional to $\left(1 / m_{j}^{2} \Gamma_{j}^{2}\right)\left[\left(O_{S_{j}}^{2} / \mathrm{v}_{S}^{2}\right)\left(O_{\eta j} \mathrm{v}_{\eta}+O_{\rho j} \mathrm{v}_{\rho}+O_{S_{j}} \mathrm{v}_{S}\right)^{2}\right]$, where the mixing angles must obey the unitary condition $\Sigma_{j} O_{S_{j}}^{2}=1$ and the VEVs are related by the constraint $\mathrm{v}_{\eta}^{2}$ $+\mathrm{v}_{\rho}^{2}+\mathrm{v}_{S}^{2}=\mathrm{v}_{E S M}^{2}=(246 \mathrm{GeV})^{2}$. As we said before once the VEV $v_{S}$ is only needed to give mass to the charged leptons, it can take small values so that the VEV-mixing-angle relation between the square brackets above can enhance the total Higgs contribution in such way that it can be still significant even for large values of the Higgs boson mass.

In this case the HSEF is given by

$$
f_{W W}=\left(O_{S 3}^{2} / \mathrm{v}_{S}^{2}\right)\left(O_{\eta 3} \mathrm{v}_{\eta}+O_{\rho 3} \mathrm{v}_{\rho}+O_{S 3} \mathrm{v}_{S}\right)^{2} .
$$

The behavior of $f_{W W}$ is shown in Fig. 1 as a function of $\mathrm{v}_{S}$ when a choice for the others parameters is made.

In Fig. 2 we show $\mathrm{S}$ and $\mathrm{B}$ for the $W W$ process for the ESM and the 3-3-1 model. There we can see that as a result of the HSEF, S is considerably enlarged for the 3-3-1 model, providing a better ratio $S / \sqrt{B}$ when comparing with the ESM. We have built these figures by considering the $s$-channel exchange of a single Higgs boson in the 3-3-1 amplitudes. Here we have taken $j=3$; however, in order to unify the notation we use $m_{3}=m_{h}$ and $\Gamma_{3}=\Gamma_{h}$. For very 


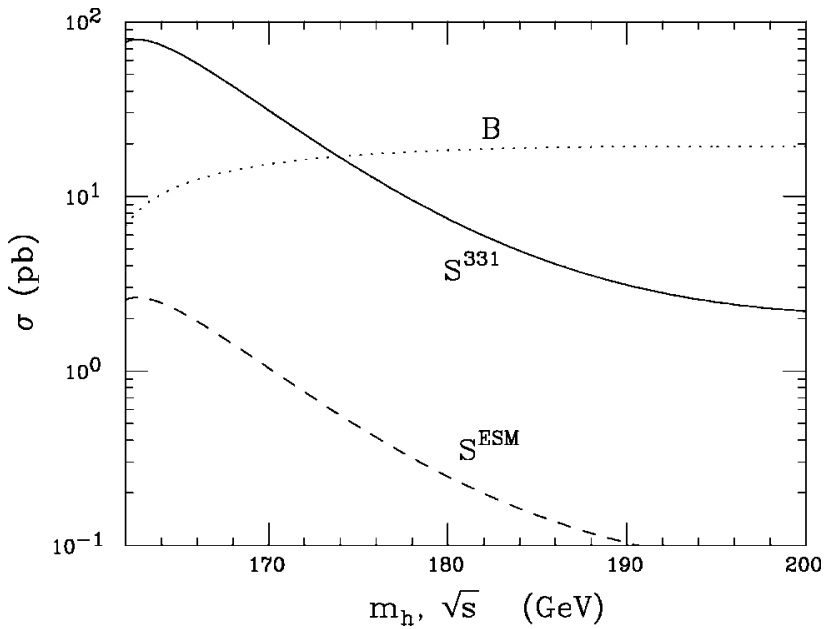

FIG. 2. $\mathrm{S}$ for the 3-3-1 model (solid line) and for the ESM (dashed line) as a function of $\sqrt{s}=m_{h}$, and B (dotted line) as a function of $\sqrt{s}$ for the $W W$ process with $\mathrm{v}_{\eta}=\mathrm{v}_{\rho}=173.8 \mathrm{GeV}, \mathrm{v}_{S}$ $=10 \mathrm{GeV}, O_{\eta 3}=0.18, O_{\rho 3}=0.085$, and $O_{S 3}=0.98$.

large values of $\sqrt{s}$ we certainly must add all other scalar contributions of the 3-3-1 model (and the boson $Z^{\prime}$ as well) in order to ensure unitarity. However, as we said before, for the values of $\sqrt{s}$ we are considering here we are assuming that the other scalars $h_{1}, h_{2}$ (and also the $Z^{\prime}$ ) are massive enough to not affect this picture. Here we have considered only the case of a real $W$-pair production to illustrate that even for a relatively massive scalar a resonance can occur in the $W W$ process. However, the enhancing factor that we have pointed out here will also occur with a virtual pair production since it is introduced by a fundamental vertex of the model.

Next we consider the $\mu^{+} \mu^{-} \rightarrow \mu^{+} \mu^{-}$process. As it is well known the Higgs signal is very small in the ESM framework provided that each vertex introduces a $m_{\mu} / \mathrm{v}_{E S M}$ factor, where $m_{\mu}$ is the muon mass and $\mathrm{v}_{E S M}=246 \mathrm{GeV}$. For this process we have the contribution of the $t$ and $s$ channels;

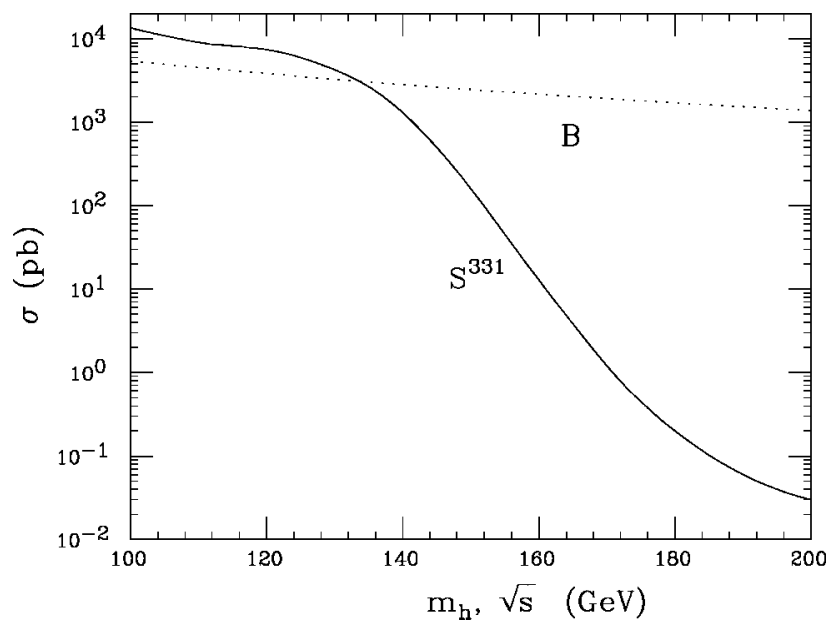

FIG. 3. S for the 3-3-1 model (solid line) as a function of $\sqrt{s}$ $=m_{h}$ and $\mathrm{B}$ (dotted line) as a function of $\sqrt{s}$ for the $\mu \mu$ process with $\mathrm{v}_{S}=10 \mathrm{Gev}$.

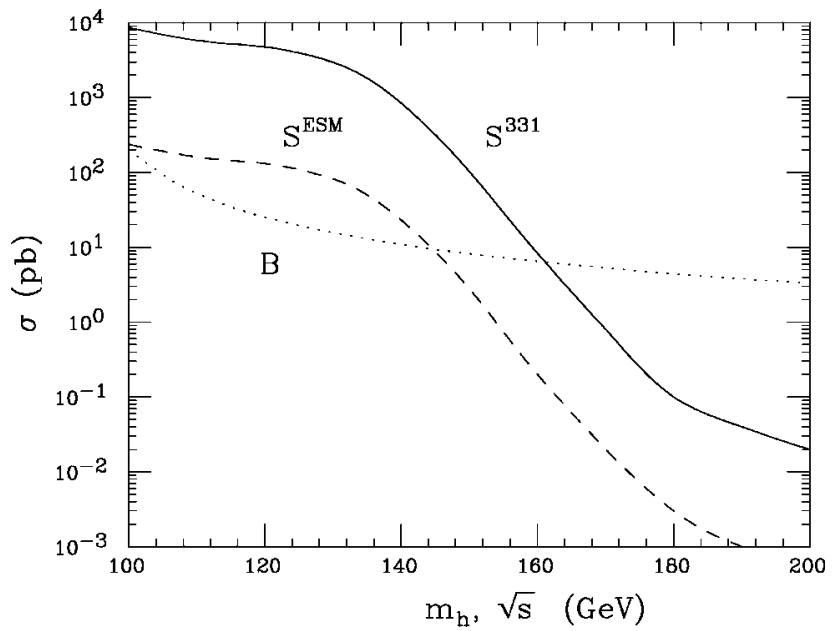

FIG. 4. S for the 3-3-1 model (solid line) and for the ESM (dashed line) as a function of $\sqrt{s}=m_{h}$ and B (dotted line) as a function of $\sqrt{s}$ for the $b b$ process with $\mathrm{v}_{S}=10 \mathrm{GeV}, \mathrm{v}_{\eta}$ $=173.8 \mathrm{GeV}, O_{S 3}=0.98$, and $O_{\eta 3}=0.18$.

however, the last one is largely dominant at the resonance. Hence the main $s$-channel Higgs contribution to $S^{E S M}$ is proportional to $\left(m_{\mu} / \mathrm{v}_{E S M}\right)^{4} /\left(m_{h}^{2} \Gamma_{h}^{2}\right)$ at the Higgs peak. Since $m_{\mu} / \mathrm{v}_{E S M}$ is an additional suppression factor and following the same argument we used before, a ESM Higgs resonance is not expected to be seen in this process. On the other hand, in the 3-3-1 model, since the Yukawa coupling is not too suppressed, the Higgs signal $\mathrm{S}^{331}$ is proportional to $\left(O_{S 3}\right)^{4}\left(m_{\mu} / \mathrm{v}_{S}\right)^{4} /\left(m_{h}^{2} \Gamma_{h}^{2}\right)$ at the resonance. As we said early, once $\mathrm{v}_{S}$ can be chosen relatively small $(10-50 \mathrm{GeV})$, the factor $\left(O_{S 3}\right)^{4}\left(m_{\mu} / \mathrm{v}_{S}\right)^{4}$ can considerably enhance the Higgssignal when comparing with the ESM one. In fact the HSEF $f_{\mu \mu}$ is given by

$$
f_{\mu \mu}=\left(O_{S 3}\right)^{4}\left(\mathrm{v}_{E S M} / \mathrm{v}_{S}\right)^{4},
$$

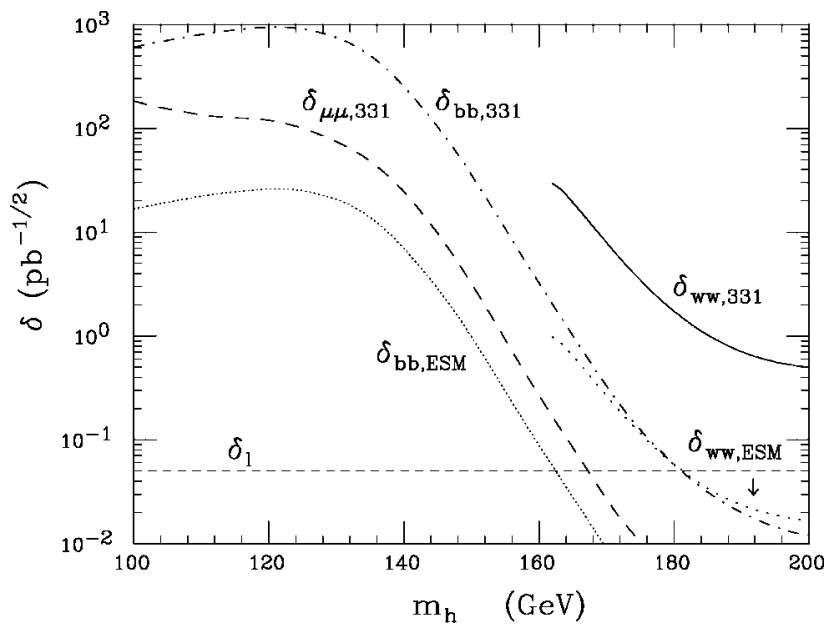

FIG. 5. The quantity $\delta=\mathrm{S} / \sqrt{\mathrm{B}}$ as a function of $m_{h}$ for the $W W$, $\mu \mu$, and $b b$ processes for the 3-3-1 model and the ESM, as indicated on the lines, for the same parameters we have used in Figs. $2-4$. The limit line $\delta_{l}=0.05 \mathrm{pb}^{-1 / 2}$ for $N_{\mathrm{S}} / \sqrt{N_{\mathrm{B}}}=5$ for $\mathcal{L}_{\mu \mu}$ $=10^{4} \mathrm{pb}^{-1} \mathrm{yr}^{-1}$. 
TABLE I. The quantities $\mathrm{S}, \mathrm{B}, N_{\mathrm{S}}, N_{\mathrm{B}}$, and the accuracies $N_{\mathrm{S}} / \sqrt{N_{\mathrm{B}}}$ and $\sqrt{N_{\mathrm{S}}+N_{\mathrm{B}}} / N_{\mathrm{S}}$ for $\sqrt{s}=m_{h}$, and for $\mathcal{L}_{\mu \mu}=10^{4} \mathrm{pb}^{-1} \mathrm{yr}^{-1}$.

\begin{tabular}{lccccccc}
\hline \hline Process & $m_{h}(\mathrm{GeV})$ & $\mathrm{S}(\mathrm{pb})$ & $N_{\mathrm{S}}$ (events) & $\mathrm{B}(\mathrm{pb})$ & $N_{\mathrm{S}}($ events $)$ & $N_{\mathrm{S}} / \sqrt{N_{\mathrm{B}}}$ & $\sqrt{N_{\mathrm{S}}+N_{\mathrm{B}}} / N_{\mathrm{S}}$ \\
\hline$b b-E S M$ & 162 & 0.138 & 1380 & 6.219 & 62190 & 5.5 & 0.18 \\
$W W-E S M$ & 180 & 0.265 & 2650 & 19.364 & 193640 & 6.0 & 0.16 \\
$\mu \mu-331$ & 167 & 2.334 & 23340 & 1984.943 & 19849430 & 5.2 & 0.19 \\
$b b-331$ & 180 & 0.123 & 1230 & 4.431 & 44310 & 5.8 & 0.17 \\
$W W-331$ & 200 & 1.153 & 11530 & 19.263 & 192630 & 26.3 & 0.04 \\
\hline \hline
\end{tabular}

and this quantity can vary from $10^{2}$ for $\mathrm{v}_{S}=50 \mathrm{GeV}$ to $10^{5}$ for $\mathrm{v}_{S}=10 \mathrm{GeV}$ assuming that $O_{S 3} \sim \mathcal{O}(1)$ as showed in Fig. 1. In Fig. 3 we show $\mathrm{S}$ and $\mathrm{B}$ as a function of $\sqrt{s}=m_{h}$ and $\sqrt{s}$, respectively. We do not quote $\sigma_{h}^{E S M}$ because it is negligible. There we can see that for relatively light Higgs boson $\sigma_{h}^{331}$ dominates the cross section, and that for $m_{h}>135 \mathrm{GeV}$ $\mathrm{S}^{331}$ goes under the B and falls rapidly to zero; however, the resulting HSEF is still enough to allow for a Higgsresonance detection in the range $m_{h} \sim 150-160 \mathrm{GeV}$ in the $\mu^{+} \mu^{-} \rightarrow \mu^{+} \mu^{-}$process. Although that enhancing factor can provide a very pronounced peak in the total cross section at the Higgs resonance for relatively light Higgs, we must remember that for this sort of mass the Higgs width is very small and this will require a very-high-resolution energy scan. As it was shown in Fig. 2, this is not the case for the $\mu^{+} \mu^{-} \rightarrow W^{+} W^{-}$process: $\mathrm{S}^{331}$ remains appreciable even for $m_{h}>170 \mathrm{GeV}$. In this case a resonance detection is much easier since for masses in this range the Higgs width is considerably large and so the energy-resolution requirements can be less stringent.

Finally we will examine the $\mu^{+} \mu^{-} \rightarrow b \bar{b}$ process. In the ESM the $b \bar{b}$ final state considerably increases the $\mathrm{S}^{E S M}$ due to the $b$-quark mass as it is shown in Fig. 4.

There we can see that, differently from the $\mu \mu$ process, the Higgs signal dominates over the background up to $m_{h}$ $<145 \mathrm{GeV}$. Although the total cross section for $b \bar{b}$ production is much lower than for $\mu^{+} \mu^{-}$, the higher ratio $\mathrm{S} / \sqrt{\mathrm{B}}$ provided by $b b$ makes this one the process to be studied at muon colliders in order to detect a relatively light Higgs resonance [1].

For the $b$ quarks the main flavor-conserving Yukawa interaction is given by $\mathcal{L}_{b}^{Y}=-\left(m_{b} / \mathrm{v}_{\eta}\right) \Sigma_{j} O_{\eta j} h_{j}^{0} \bar{b} b$, Hence, the pure $s$-channel Higgs contribution to $b \bar{b}$ at the resonance is proportional to $\left(1 / m_{h}^{2} \Gamma_{h}^{2}\right)\left(O_{S 3} O_{\eta 3}\right)^{2}\left(m_{\mu} m_{b} / \mathrm{v}_{S} \mathbf{v}_{\eta}\right)^{2}$, In this case, for $\sqrt{s}=m_{h}$, the $\operatorname{HSEF} f_{b \bar{b}}$ is given by

$$
f_{b \bar{b}}=\left(O_{S 3} O_{\eta 3}\right)^{2}\left(\frac{\mathrm{v}_{E S M}^{2}}{\mathrm{v}_{S} \mathrm{v}_{\eta}}\right)^{2} .
$$

As before this factor allows the possibility of having an enhancement in the pure Higgs amplitude as showed in Fig. 1.
In Fig. 5 we show the quantity $\delta$ defined above for the three processes and the two models considered here. We omit $\delta_{\mu \mu}$ for the ESM because it is negligible. From Fig. 5 we can see that in the ESM framework, and assuming that $N_{\mathrm{S}} / \sqrt{N_{\mathrm{B}}} \geqslant 5$ is necessary for detection, the $b b$ process is able to detect Higgs resonances for $m_{h}$ up to $\sim 162 \mathrm{GeV}$. Beyond this Higgs boson mass, only the $W W$ process provides a $\delta_{W W}$ large enough to allow for detections for $m_{h}$ up to $\sim 180 \mathrm{GeV}$. In the same way, Fig. 5 also shows that in the 3-3-1 model framework the $\mu \mu$ process is sensitive to detect Higgs resonances for $m_{h}$ up to $\sim 167 \mathrm{GeV}$, the $b b$ process up to $\sim 180 \mathrm{GeV}$, and that the $W W$ is the only process able to investigate Higgs resonances for $m_{h}$ in the heavy-mass range $m_{h}>200 \mathrm{GeV}$. Here we are using the luminosity $\mathcal{L}_{\mu \mu}=10^{4} \mathrm{pb}^{-1} \mathrm{yr}^{-1}$ so that in order to be compatible with the $N_{\mathrm{S}} / \sqrt{N_{\mathrm{B}}} \geqslant 5$ requirement, we must have $\delta \geqslant 5$ $\times 10^{-2} \mathrm{pb}^{-1 / 2}$. This limit line denoted by $\delta_{l}$ is also shown in Fig. 5. The values given above concerning the 3-3-1 model should be taken as indicative only once they depend on a set of parameters that are a priori free. In Table I we summarize the above results given $\mathrm{S}$ and $\mathrm{B}$, and the corresponding $N_{\mathrm{S}}$ and $N_{\mathrm{B}}$, and accuracies, for these representative Higgs boson masses.

The existence of a Higgs resonance far above the ESM limit will indicate at least that that scalar no longer belongs to the minimal ESM and that new physics must be brought in. Although this resonance may exist in a multi-Higgsboson $\mathrm{SU}(2)_{L} \otimes \mathrm{U}(1)_{Y}$ model, this is only possible by extending the symmetry with a discrete one. On the other hand, in the 3-3-1 model the fact that the sextet couples only to leptons is just a consequence of the representation content in the lepton sector in the minimal model. Hence, the scalar resonance seems more natural in last model than in multiHiggs-boson extensions of the electroweak standard model.

This work was supported by Fundação de Amparo à Pesquisa do Estado de São Paulo (FAPESP), Conselho Nacional de Ciência e Tecnologia ( $\mathrm{CNPq})$, and Programa de Apoio a Núcleos de Excelência (PRONEX). One of us (C.P.) would like to thank Coordenadoria de Aperfeiçoamento de Pessoal de Nível Superior (CAPES) for financial support. 
[1] V. Barger, M. S. Berger, J. F. Gunion, and T. Han, Phys. Rev. Lett. 75, 1462 (1995); Phys. Rev. B 286, 1 (1997).

[2] F. Pisano and V. Pleitez, Phys. Rev. D 46, 410 (1992); P. Frampton, Phys. Rev. Lett. 69, 2889 (1992).

[3] P. A. Baikov et al., in Proceedings of X Workshop on High Energy Physics and Quantum Field Theory (QFTHEP-95), Moscow, 1994, edited by B. Levtchenko and V. Savrin, hep-ph/9701412, p. 101; E. E. Boos, M. N. Dubinin, V. A. Ilyin, A. E. Pukhov, and V. I. Savrin, “COMPHEP: Special- ized Package for Automatic Calculations of Elementary Particle Decays and Collisions," SNUTP-94-116, 1994, hep-ph/9503280.

[4] B. Kamal, W. J. Marciano, and Z. Parsa, presented at the Workshop on Physics at the First Muon Collider and at the Front End of the Muon Collider, Batavia, Illinois, 1997, pp. 657-662, hep-ph/9712270.

[5] M. D. Tonasse, Phys. Lett. B 381, 191 (1996). 